\title{
Erratum: Applying clustering to statistical analysis of student reasoning about two-dimensional kinematics [Phys Rev. ST Phys. Educ. Res. 3, 020107 (2007)]
}

\author{
R. Padraic Springuel, ${ }^{*}$ Michael C. Wittmann, and John R. Thompson \\ (Received 9 July 2009; published 9 November 2009) \\ DOI: 10.1103/PhysRevSTPER.5.029902 \\ PACS number(s): 01.40.Fk, 01.40.gf, 99.10.Cd
}

\section{INTRODUCTION}

In our paper reported previously in this journal, we explored how cluster analysis, a method from data mining used to find natural groupings in data, could be used to categorize the responses given by students on a free-response question about acceleration in two dimensions. In the process of preparing to expand on that work, however, we discovered that our analysis was both incorrect and incomplete. We were incorrect in that we used default settings in our software package and thereby misidentified the distance measure used. We have since determined that this distance was not appropriate for our data coding. Furthermore, we were incomplete in that we were not sufficiently rigorous in our definition of groups of student responses. We have corrected both of these issues.

\section{MISIDENTIFIED DISTANCE}

In our original paper, we used a default setting in a software package for the distance measure between two responses. In the documentation provided by de Hoon et al. ${ }^{1}$ for their software package, this distance function is referred to as the "Euclidian distance." However, the distance, $d$, is defined mathematically as follows:

$$
d=\frac{1}{n} \sum_{i=1}^{n}\left(x_{i}-y_{i}\right)^{2}
$$

Only those terms are included in the summation for which both $x_{i}$ and $y_{i}$ are present. The denominator $n$ is chosen accordingly ${ }^{1,2}$ (p. 6).

This differs from the traditional definition of the Euclidian distance in two important ways.

First the factor $\frac{1}{n}$ serves to account for the fact that different data points may be missing different numbers of $x_{i}$ and $y_{i}$. While our data did not contain any such situations (and thus $n$ was constant), this factor effectively normalized the resultant distances because our data was binary. This was noted in our paper.

Second, it fails to take the square root of the sum, meaning that this is a squared Euclidian distance, not a Euclidian distance. It was this aspect of the distance that we failed to notice in our paper. All references in our paper to a Euclidian distance should thus be replaced by a reference to a squared Euclidian distance.

\section{CHOOSING AN APPROPRIATE DISTANCE}

The wrong definition of Euclidean distance is overshadowed by the inappropriate choice of the Euclidean distance itself. In our previous work we gave three reasons for choosing the unweighted, normalized, (squared) Euclidian distance:

(1) it is calculationally equivalent to the distance measure used by Montenegro, Aubrecht, and Bao, ${ }^{3}$ (2) it is readily available in the software package selected for this study, (3) it is easy to compute by hand if necessary to check the computer calculations as any distance between two student responses will be [...] an integer divided by the number of dimensions in the answer vector. ${ }^{4}$

While these reasons seemed acceptable to us at the time, none are based on considerations of the data or coding scheme. Different types of data have different mathematical properties. ${ }^{5}$

For example, the squared Euclidean distance is appropriate for interval data. For interval data, such as temperature or grades, we can determine whether values are equal or unequal, greater than or less than other values. We can also carry out the mathematical processes of addition and subtraction. To make our data appear to be interval data (and thus allow the squared Euclidean distance function to work), we had to give numerical values to the two states (presence $=1$, absence $=0$ ) and assume that the difference between these numerical values represented a measure of the difference between two data points in that category. This was inappropriate.

Our coding scheme described students' drawn arrows by a series of binary categories which indicated the presence or absence of certain features of the arrow. As a result, we had purely asymmetric nominal data. Such data are defined by two characteristics: a set of distinct possible responses and the presence within that set of a single option which provides no usable information. ${ }^{6}$ For this reason asymmetric nominal data does not always give us useful information, and that information is 
confined to being in one of the predefined categories. Mathematically this means that we can establish equality ( $=$ ) and inequality $(\neq)$ between two responses, but not all the time. Conceptually, students who share a response are thought of as equal, but students who are both lacking a response cannot be thought of as equal, because we do not know enough about why a given response is missing. Therefore, we must discount matches of "absent" from determining the distance between two data points.

Recall that in our analysis, a student's response was presented as a student answer vector (SAV), with $N$ elements. A Jaccard distance $\left(d^{J}\right)$ would be the most appropriate way to determine the distance between two SAV:

$$
d_{\vec{A} \vec{B}}^{J}=\frac{\sum_{i=1}^{N}\left(1-\delta_{A_{i} B_{i}}\right)}{N-\sum_{i=1}^{N} \delta_{A_{i} \varnothing} \delta_{B_{i}} \varnothing}
$$

where $\vec{A}$ is the SAV for student A (with $A_{i}$ being the coding on the $i^{\text {th }}$ category), $\vec{B}$ is similarly defined for student $\mathrm{B}, N$ is the number of dimensions in $\vec{A}$ and $\vec{B}$, and $\varnothing$ is used as a short hand notation for an absence coding.

The Jaccard and squared Euclidian distance differ only in the denominator, because the numerators are equal for our data. For arbitrary $x$ and $y$ which are restricted to taking on values of 0 and 1, the numerators of the squared Euclidian distance and the Jaccard distance are identical because $(x-y)^{2}=1-\delta_{x y}$. The Jaccard distance discounts absence matches in the denominator (the $\sum_{i=1}^{N} \delta_{A_{i} \varnothing} \delta_{B_{i} \varnothing}$ term). The Jaccard distance typically increases for students whose responses are coded with many absent values of $x$ or $y$. Mathematically, we would expect the Jaccard distance between two students' responses to be equal to or larger than the squared Euclidian distance between them, as shown by the following:

$$
\begin{gathered}
\frac{\sum_{i=1}^{N}\left(1-\delta_{A_{i} B_{i}}\right)}{N-\sum_{i=1}^{N} \delta_{A_{i} \varnothing} \delta_{B_{i} \varnothing}} \geqslant \frac{\sum_{i=1}^{N}\left(A_{i}-B_{i}\right)^{2}}{N} \\
d_{\vec{A} \vec{B}}^{J} \geqslant d_{\vec{A} \vec{B}}^{S E}
\end{gathered}
$$

where our symbols are defined as they were for Eq. (3).

\section{TESTING DATA WITH NEW DISTANCE MEASURES}

Unfortunately, because any pair of SAVs might have a different number of absence matches, there is no way to predict exactly how much greater the Jaccard distance is than the squared Euclidian distance. There is no guarantee that it will be a monotonic change to go from using squared Euclidian distances to Jaccard distances (i.e., Student A may be closer to Student B than to Student C under the squared Euclidian distance, but closer to Student C than to Student B under the Jaccard distance). As a result, we cannot assume that the results of the cluster analysis we reported are an accurate reflection of the information present in the data. We must check the previous results.

We reran the cluster analysis using the Jaccard distance. Since the code implemented by de Hoon et al. ${ }^{1}$ does not provide for the Jaccard distance as one of the options, new code was written to perform the agglomerative hierarchical clustering using either the Jaccard distance or the Euclidian distance. ${ }^{7,8}$

\section{A. Defining groups rigoruously}

Once the two versions of the hierarchical clustering were performed, it was necessary to find the groups in the resultant trees. Previously, we had identified groups mostly by eye in our paper. When labeling the groups, deciding how many students were in them, and finding the centroids (i.e., the "typical" answer for the group), we relied on de Hoon et al.'s "cuttree" and "getclustercentroids" algorithms. These, when nested, find the centroids for a given horizontal slice of the tree. This led to mismatches in the identified characteristics that described a group and the identification to how many students were in a group.

To correct this error, we decided to tighten up the definition of a group. To that end, we came up with three criteria for what constituted a group: (1) a group had to have at least one category which we would classify as a dominant characteristic (i.e., all students were coded as "present" on this category); (2) the population of the group had to be at least 1/30 of the entire population; (3) a group could not be a subset of another group (i.e., groups were defined as high on the tree as possible). Furthermore, we defined a subgroup when it was possible to follow the tree downwards away from a group and find a "division" or set of "divisions" which resulted in two or more paths which still met criteria 2. ${ }^{9}$ Finally, we defined a deadend as a place on the tree where criteria 2 and 3 were met, but criteria 1 was not. This leads to a slight reorganization of the 
TABLE I. Groups found by the clustering algorithm.

\begin{tabular}{|c|c|c|c|c|}
\hline $\begin{array}{l}\text { Diagram } \\
\text { Identifier }\end{array}$ & $\mathrm{Name}^{\mathrm{a}}$ & $\mathrm{N}$ & Dominant Characteristics & Prominent Characteristics \\
\hline I & No C Arrow & 106 & "No Arrow" at point C & - \\
\hline Ia & Tangent No Arrow & 45 & as I & "Tangent" at point B \\
\hline $\mathrm{Ib}$ & CtD No Arrow & 32 & $\begin{array}{l}\text { as I plus "Connect-the-Dots at point } \\
\text { B }\end{array}$ & - \\
\hline Ic & Blanks & 29 & as I & - \\
\hline II & & 59 & "Tangent" at point B & "Tangent" at point $\mathrm{C}$ \\
\hline IIa & Pure Tangent & 17 & "Tangent" at points B and C & - \\
\hline $\mathrm{IIb}$ & & 13 & as II & as II plus "Centrifugal" at point B \\
\hline IIc & & 17 & $\begin{array}{l}\text { as II plus "Tangent" and "Cen- } \\
\text { tripetal" at point C }\end{array}$ & - \\
\hline IId & Correct and Tangent & 12 & $\begin{array}{l}\text { as II plus "Centripetal" at point B } \\
\text { and "Tangent" at point C }\end{array}$ & - \\
\hline III & Tangent Zero & 41 & "0 Arrow" at point $\mathrm{C}$ & - \\
\hline IIIa & & 27 & as III & "Tangent" at point B \\
\hline IIIb & & 14 & as III & 一 \\
\hline IV & Correct or Circular & 32 & "Centripetal" at point $\mathrm{C}$ & $\begin{array}{l}\text { "Tangent" and "Centripetal" at } \\
\text { point B }\end{array}$ \\
\hline $\mathrm{V}$ & Connect-the-Dots & 22 & "Connect-the-dots" at point $\mathrm{C}$ & "Connect-the-dots" at point B \\
\hline
\end{tabular}

${ }^{a}$ This column refers to the names assigned to the groups in the original paper. Their match to the new group structure is based on Table II.

identifiers from what was presented in our paper. Table I reflects the application of these revised definitions to the new clustering algorithm.

\section{B. Comparison to original results}

To help determine how much the above-mentioned corrections changed the results of the cluster analysis process, we devised two representations which would help us compare the two results.

The first representation is presented in Fig. 1 and shows the dendrogram representations of the old and new clustering solutions next to each other. As in the original paper, we have removed any branch from the dendrogram which contains less than $1 / 30$ of the total population. However, instead of showing the original group labels, which were arbitrarily applied and didn't necessarily match numbers to centroids, we used the new definitions for what constitutes a group, subgroup, and deadend instead. This allowed us to construct our second representation, Table II, which shows where the students in one clustering solution fall in the other.
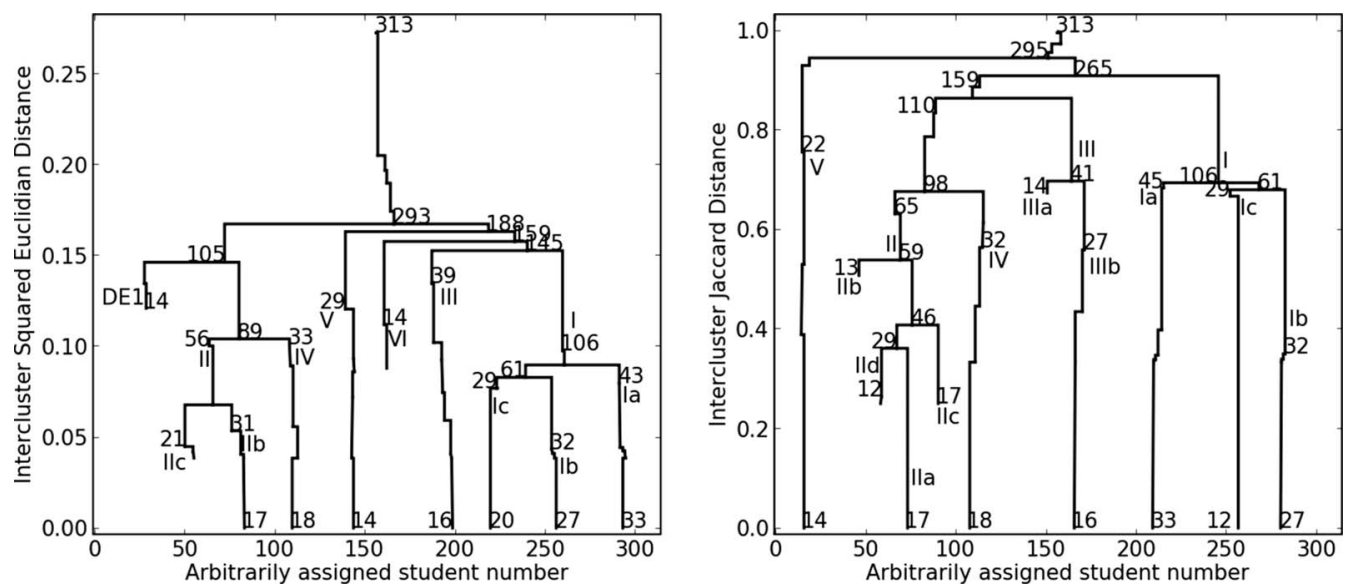

FIG. 1. The graph on the right shows the results of the cluster analysis with the square Euclidian distance (i.e., a reproduction of Fig. 4 from the original paper, except with the new group, subgroup, and deadend numbering applied), while the one on the left shows the results of the cluster analysis with the Jaccard distance. Note that a student's position along the x-axis is not necessarily consistent between the two graphs. 
TABLE II. Cross comparison of groups found using the Jaccard (down) and squared Euclidian (across) distances. Cell values of "0" have been omitted for the sake of clarity. $N=313$.

\begin{tabular}{|c|c|c|c|c|c|c|c|c|c|c|c|c|c|}
\hline & DE1 & Noise & I & $\mathrm{Ia}$ & $\mathrm{Ib}$ & Ic & II & IIa & IIb & III & IV & $\mathrm{V}$ & VI \\
\hline Noise & 6 & 25 & & & & & 1 & 1 & & 1 & 3 & 9 & 8 \\
\hline I & & & 106 & 43 & 32 & 29 & & & & & & & \\
\hline Ia & & & 45 & 43 & & & & & & & & & \\
\hline $\mathrm{Ib}$ & & & 32 & & 32 & & & & & & & & \\
\hline Ic & & & 29 & & & 29 & & & & & & & \\
\hline II & 8 & & & & & & 51 & 30 & 21 & & & & \\
\hline IIa & & & & & & & 17 & 17 & & & & & \\
\hline $\mathrm{IIb}$ & 8 & & & & & & 5 & 5 & & & & & \\
\hline IIc & & & & & & & 17 & 8 & 9 & & & & \\
\hline IId & & & & & & & 12 & & 12 & & & & \\
\hline III & & & & & & & & & & 38 & & & 3 \\
\hline IIIa & & & & & & & & & & 27 & & & \\
\hline IIIb & & & & & & & & & & 11 & & & 3 \\
\hline IV & & 3 & & & & & & & & & 29 & & \\
\hline V & & 2 & & & & & & & & & & 20 & \\
\hline
\end{tabular}

Taken together, the two representations show that while our descriptions of the groups have been changed by the corrections described above, the students are actually falling into very similar groups.

\section{RE-INTERPRETATION OF GROUPS}

\section{A. Group I}

The descriptions of this group and its subgroups remain largely unchanged. Looking at Table II it is easy to see that group I and its subgroups match up perfectly between the two clustering solutions (originally identified as no C arrow, tangent no arrow, CtD no arrow, and blanks). However, the new definitions for group, subgroup, and deadend require one minor correction. While we had previously identified no arrow at point $B$ as a dominant characteristic for subgroup Ic, only somewhere between 50 and $75 \%$ of the population of this subgroup actually did this. This means the characteristic is not even a prominent characteristic, even though it is still important enough to be noted (and thus justifying the retention of the name blanks).

\section{B. Group II}

This group has many changes from the original group. Originally identified as pure tangent and correct and tangent (subgroups IIb and IIc), our redefinition of what constituted a group led to these two subgroups being combined to form a group while the other subgroup was sectioned off into its own group (see group IV, below). As a new group, this group has tangential components at point $B$ as a dominant characteristic and at point $C$ as a prominent characteristic. We also see that under our corrections, this group has not two, but four subgroups.

Subgroup IIa promotes tangential components at point $C$ to a dominant characteristic and is made up of identical students who just had tangential components at both points. These are the students that we think are most likely exhibiting a confusion between velocity and acceleration. It matches well to the majority of subgroup IIa on the squared Euclidian side.

Subgroup IIb promotes centrifugal components at point $B$ to a prominent characteristic, taking some students from what we now identify as Deadend 1 and thus contributing to the demise of that feature from the Jaccard clustering solution. It also uses some of the students from squared Euclidian's subgroup IIa. While these students seem to be exhibiting a velocity confusion, the fact that the vast majority of them have a centrifugal component at point $B$ gives us pause in applying that label to them. As with Deadend 1, these students may be invoking some idea of centrifugal force in drawing their arrows at point $B$, but since less than $50 \%$ have centrifugal components at point $C$, the velocity confusion interpretation is far less probable. Further research is necessary here to determine what these students are doing.

Subgroup IIc promotes tangential components and centripetal components at point $C$ to dominant characteristics. Fifty to $75 \%$ of the students also have a centripetal component at point $B$, explaining why it splits across the two subgroups on the squared Euclidian side, and also hinting at what these students might be doing. With the centripetal component at point $B$ the acceleration vectors look very much like what the velocity vector will look like a short while later in the motion. These students may then be "leading" the motion with the acceleration vector. 
Subgroup IId promotes centripetal components at point $B$ and tangential components at point $C$ to dominant characteristics. Together with about half of IIc, it corresponds to subgroup IIb in the squared Euclidian clustering solution. As in the original paper, this group of students is doing something so unexpected that we are unable to hypothesize as to what they might be doing.

\section{Group III}

The dominant and prominent characteristics of this group changed. Originally identified as tangent zero (group III) it should first be noted that our corrections have eliminated tangent arrows at point $B$ as a dominant characteristic for this group (i.e., something every student did). The only dominant characteristic for this group is 0 arrows at point $C$. It is not until the subgroup level (something we did not see in the original paper) that we see tangent arrows at point $B$ enter into the picture and then only as a prominent characteristic of subgroup IIIa. Subgroup IIIb consists of those students who did not follow this pattern.

\section{Group IV}

This group should be reclassified as simply correct. In our original paper, this group was identified as correct or circular, a subgroup of components (IIa), but our redefinition of what constituted a group and a subgroup prompted a promotion to full group status. Furthermore, the formal definitions changed the identification of dominant and prominent characteristics. Arrows with a centripetal component at point $C$ is a dominant characteristic for this group while arrows with a centripetal component at point $B$ is only a prominent characteristic. Tangential components at point $B$ also appear as a prominent characteristic. However, tangential components for arrows at point $C$ appear in less than $10 \%$ of the student responses.

\section{E. Group V}

Our interpretation of the group remains unchanged. In our original paper, this group was identified as connect-the-dots (group IV). After applying our corrections, we see that a connect-the-dots type arrow at point $C$ is a dominant characteristic, while at point $B$ this kind of arrow is a prominent characteristic.

\section{F. "Missing" groups}

Our original paper identified two more groups, curve following and centrifugal which are not observed after our corrections are made. The branches of the dendrogram along which these groups can be found start out very small (14 and 16 students, respectively) and they don't actually have dominant characteristics at their beginning. Thus, by our new, more rigorous definition, they are no longer groups for this pilot data set. A larger and more complete data set would help to determine if curve following and centrifugal qualify as groups or not in a more general sense.

\section{CONCLUSIONS}

The errors in our previous paper arose from two unsupported choices. Our choice of prepackaged software had the consequence of applying an inappropriate distance measure. This affected how clusters were grouped. This was not observed sooner because squared Euclidean and Jaccard distances are quite similar for our data, and thus the changes were small. Also, our choice for group definitions was $a d$ hoc and prone to errors. Creating a rigorous definition of groups uncovered several errors in our previous descriptions of the groups. After correcting these errors, however, we find that the method of cluster analysis is still a viable and powerful tool for grouping student responses, and that rigorous choices of distance measure and grouped responses gives valuable insight into what students in our classes are doing as they answer our questions. 
*R.Springuel@umit.maine.edu

${ }^{1}$ M. de Hoon, S. Imoto, and S. Miyano, The C clustering library, http://bonsai.ims.u-tokyo.ac.jp/mdehoon/software/cluster/cluster.pdf (2008).

${ }^{2}$ The mathematical notation in Eq. (1) differs slightly from the notation we will use later in this paper. In our notation, this equation would appear as:

$$
d_{\vec{A} \vec{B}}^{S E}=\frac{1}{N} \sum_{i=1}^{N}\left(A_{i}-B_{i}\right)^{2}
$$

Where the superscript $S E$ serves to identify the distance as a squared Euclidian and definitions for the other terms follow Eq. (3)).

${ }^{3}$ M. Montenegro et al., (2006).

${ }^{4}$ The missing text here is "the square root of" and was included in the original because of our misidentification of the distance. It is left out of the quote here to reflect our corrected understanding of the distance.

${ }^{5}$ We intend to discuss this matter in more detail in a future publication.

${ }^{6}$ L. Kaufman and P. J. Rousseeuw, Finding groups in data: An introduction to cluster analysis, Wiley series in probability and mathematical statistics: Applied probability and statistics (Wiley, 1990).

${ }^{7}$ R. P. Springuel, Python module for cluster analysis, URL http://www.umit.maine.edu/ r.springuel/FOV18-000CCFE8/ cluster.zip.

${ }^{8}$ The code is actually more general than this and forms a complete alternative to and expansion on The C Clustering Library. Reference 7 points to the code.

9 "Division" is placed in quotation marks because the trees are formed through a series of joinings. Said joinings can be considered divisions only in the sense that the distance order of the tree can be reversed from that which created it. Since this is a much harder process to code than agglomeration, this remains a theoretical reversal. 